\title{
La Preparación de los Maestros para Estimular la Socialización De los Educandos con Autismo en Condiciones de INCLuSión ${ }^{1}$ The Preparation of School Teachers to Stimulate the Socialization of STUDENTS WITH AUTISM IN CONDITIONS OF INCLUSION
}

\author{
Osvaldo Hernández GONZÁLEZ² \\ Caroll Alejandra Schilling LARA ${ }^{3}$ \\ Rossana GÓMEZ-CAMPOS 4 \\ Marco COSSIO-BOLAÑOS 5 \\ Rosario Elena Spencer CONTRERAS ${ }^{6}$
}

\begin{abstract}
RESUMEN: Los maestros de educación general han expresado su preocupación por la inclusión de educandos con Trastornos del Espectro Autista, debido a la falta de habilidades sociales de los niños, comportamientos estereotipados e intereses restringidos. El objetivo de este estudio es describir y comparar el estado actual de la preparación de los maestros de primaria para estimular la socialización de los educandos con Trastornos del Espectro Autista en escuelas inclusivas. A la luz de una metodología cuantitativa se realizó un estudio descriptivo-comparativo de corte transversal. Se estudiaron 74 maestros (52 mujeres y 22 hombres) encargados de cumplir con el rol docente-educativo de atender a los educandos con Trastornos del Espectro Autista en condiciones de inclusión en una provincia occidental de Cuba. El rango de edad oscila entre 22 y 57 ańos. Se aplicó un cuestionario y una Ficha de Observación previamente validados para medir la preparación teórica-metodológica y práctica de los maestros. Los resultados muestran que los maestros presentan dificultades para estimular la socialización de los educandos con autismo en escuelas inclusivas. Asimismo, el grado académico, la experiencia en espacios educacionales inclusivos y el área disciplinar resultaron ser variables sociodemográficas que determinan la preparación de los maestros para cumplir con esta tarea educativa. Estos resultados aportan información clave para diseñar estrategias y planes de acción, tanto en la formación inicial como en la práctica cotidiana, en aras de potenciar las competencias teóricas y prácticas de los maestros que asumen este desafío.
\end{abstract}

PALABRAS-CLAVE: Educación Especial. Autismo. Inclusión Educativa. Formación Docente.

\begin{abstract}
Basic Education teachers have expressed concern about the inclusion of learners with Autism Spectrum Disorder, due to children's lack of social skills, stereotypical behaviors, and restricted interests. The objective of this study was to describe and compare the current state of preparation of Elementary School teachers to stimulate the socialization of students with Autism Spectrum Disorder in inclusive schools. In the light of a quantitative methodology, a descriptive-comparative cross-sectional study was carried out. Seventy-four teachers ( 52 women and $22 \mathrm{men}$ ) in charge of fulfilling the teaching-educational role of attending to students with Autism Spectrum Disorder in conditions of inclusion in a Western province of Cuba were studied. The age ranged from 22 to 57 years. A previously validated questionnaire and Observation Sheet were applied to measure teachers' theoreticalmethodological and practical preparation. The results show that teachers have difficulties in stimulating the socialization of learners with autism in inclusive schools. Likewise, the academic degree, the experience in inclusive educational spaces and the disciplinary area turned out to be sociodemographic variables that determine the preparation of the teachers to fulfill this educational task. These results provide key information to design strategies and action plans, both in initial training and in daily practice, in order to enhance the theoretical and practical skills of teachers who take on this challenge.
\end{abstract}

KEYWORDS: Special Education. Autism. Educational inclusion. Teacher training.

\footnotetext{
${ }^{1}$ https://doi.org/10.1590/1980-54702021v27e0197

${ }^{2}$ Magíster en Educación Especial y Psicopedagogía. Estudiante de Doctorado en Ciencias Humanas en la Universidad de Talca. Talca/Chile. E-mail: osvaldo.hernandez@utalca.cl. ORCID: https://orcid.org/0000-0002-1319-6167

${ }^{3}$ Doctora en Ciencias de la Educación. Docente e investigadora en la Facultad de Educación de la Universidad Católica del Maule. Talca/Chile. E-mail: cas11975@gmail.com. ORCID: https://orcid.org/0000-0002-9047-6306

${ }^{4}$ Doctora en Educación Física. Docente e investigadora en la Facultad de Educación de la Universidad Católica del Maule. Talca/ Chile. E-mail: rossaunicamp@gmail.com. ORCID: https://orcid.org/0000-0001-6509-5707

${ }^{5}$ Doctor en Ciencias del Deporte. Docente e investigador en la Facultad de Educación de la Universidad Católica del Maule. Talca/ Chile. E-mail: mcossio@ucm.cl. ORCID: https://orcid.org/0000-0001-7230-9996

${ }^{6}$ Doctora en Psicología Clínica. Docente e investigadora en la Facultad de Psicología de la Universidad de Talca. Talca/Chile. E-mail: rspencer@utalca.cl. ORCID: https://orcid.org/0000-0002-2626-9257
} 


\section{INTRODUCCIÓN}

Los Trastornos del Espectro Autista (TEA) se caracterizan por alteraciones cualitativas en las habilidades sociales y la comunicación verbal y no verbal, la presencia de intereses y comportamientos estereotipados y restringidos, así como también trastornos del sueño y de la ingestión de alimentos, rabietas, agresiones o autoagresiones (American Psychiatric Association [APA], 2013). Los síntomas aparecen en las primeras edades del desarrollo, y en proporciones variables, alrededor del $1 \%$ de la población en distintos lugares del mundo presenta esta condición neurocomportamental (Christensen et al., 2016). Las principales causas del TEA incluyen una amplia gama de alteraciones genéticas y se considera el espacio socio-cultural como una variable clave para su pronóstico, pero a ciencia cierta su identidad aún no es bien comprendida (Vorstman et al., 2017).

En los últimos lustros, la prevalencia del TEA ha aumentado de manera exponencial (Smiley et al., 2018; Tromans et al., 2018). Este aumento de la prevalencia (Xu et al., 2019) ha sucedido en medio de un escenario educacional inclusivo que pretende dar respuesta a la diversidad en el aula, promovido por la Conferencia Mundial de Jomtien sobre Educación para Todos (Organización de las Naciones Unidas para la Educación, la Ciencia y la Cultura [UNESCO], 1990), la Declaración de Salamanca (Organización de las Naciones Unidas para la Educación, la Ciencia y la Cultura [UNESCO], 1994) y la Convención de las Naciones Unidas sobre los Derechos Humanos de las Personas con Discapacidad (Naciones Unidas [ONU], 2006). Esto ha propiciado que en la actualidad muchos educandos con esta condición del neurodesarrollo participen cada vez más en las actividades que se llevan a cabo en las escuelas convencionales. Esta realidad ha dado paso a su vez a un flujo importante de investigación que busca dar cuenta de las experiencias de los docentes y de los estudiantes con TEA en este nuevo modelo educativo (Frombonne, 2018; Snyder \& Dillow 2013).

Muchas investigaciones apuntan a que la inclusión de las personas con TEA en las escuelas principales contribuye a potenciar su desarrollo personal y social, puesto que para cambiar el curso del autismo hacia una trayectoria más adaptativa y funcional del desarrollo es vital que estos participen en espacios educacionales y culturales que estimulen su socialización (Lindsay et al., 2013; McCurdy \& Cole, 2013; Prizant, 2015). En una reciente investigación llevada a cabo por Garrad et al. (2018), se subraya que es importante comprender las perspectivas de los maestros que tienen dentro de su encargo social atender a educandos con TEA en aulas principales, como un paso capital para avanzar hacia la inclusión exitosa.

En ese sentido, varios estudios han mencionado que los maestros se muestran receptivos con la responsabilidad de gestionar y conducir una sala de clase inclusiva que responda también a las necesidades y motivaciones fundamentales de los educandos con TEA (Roberts \& Simpson, 2016; Rodden et al., 2019). Estas actitudes positivas hacia la inclusión cumplen un papel capital, puesto que la actividad pedagógica que realizan los maestros puede favorecer la implementación de prácticas inclusivas o, por el contrario, puede constituirse en una barrera para el aprendizaje y la participación del alumnado con TEA (Carrington et al., 2016; Chung et al., 2015). Sin embargo, otras investigaciones han puesto de relieve que los maestros perciben la inclusión de los educandos con TEA en las aulas principales como un problema, debido al comportamiento disruptivo e inusual de los mismos, la escasez de tiempo y los recursos para brindarle el apoyo que necesitan (Anglim et al., 2018). Todo ello podría reducir las expectativas 
de los maestros sobre lo que saben y lo que pueden hacer en estas condiciones prácticas, con consecuencias directas en su labor social y en el rendimiento de los estudiantes con autismo (Ashburner et al., 2010).

En la nación cubana, para garantizar la educación de toda la población, las autoridades políticas y los agentes de socialización han colaborado de manera sistemática y propositiva en la planificación de disímiles servicios y modalidades de enseñanza que buscan potenciar la igualdad de oportunidades de aprendizaje (Serrano, 2017). En este contexto las actividades educativas se estructuran teórica y metodológicamente de acuerdo a los postulados de la escuela histórico-cultural de Vigotsky, la cual se caracteriza por un estilo pragmático e integrador que considera los intercambios sociales como determinantes en el desarrollo de la personalidad. Independiente de los esfuerzos realizados y los logros alcanzados, la inclusión de educandos con TEA continúa siendo un reto para el sistema educativo cubano, especialmente para los maestros.

Con lo dicho hasta aquí, es posible apuntar que la preparación que posean los maestros para trabajar con educandos con TEA, es un aspecto esencial que les permitirá interactuar con sus problemas cognitivos, emocionales y volitivos y encauzar de manera eficiente y eficaz los estímulos educativos necesarios para potenciar su socialización (Anglim et al., 2018; Chen et al., 2017; Soto-Chodiman et al., 2012). Sin embargo, la mayoría de los estudios se centra en el componente actitudinal (Garcés \& Zambrano, 2018; Garrad et al., 2018; Low et al., 2018; Srivastava et al., 2017), pero hay relativamente poca investigación centrada en la preparación de los maestros entendiéndola como el resultado global de un proceso que está en constante transformación en un amplio escenario de colaboración grupal (Castillo, 2004). En este sentido, la preparación de los maestros puede determinar sus interacciones diarias con los estudiantes con TEA, así como también con sus padres y provocar un impacto en los mismos (Chung et al., 2015; Lozic, 2014).

El maestro deberá responder a las demandas de cada uno de sus estudiantes, así como del contexto familiar y social donde se desarrolla (García et al., 2016). Desde esta perspectiva, la preparación de los maestros trasciende las líneas de la enseńanza de contenidos y abarca todas las dimensiones del trabajo educativo, lo cual incluye la orientación de la familia y la respuesta a problemas emocionales dentro del aula para favorecer la socialización del educando con TEA (Campos, 2010). Por lo tanto, existe una clara necesidad de investigación de pasar de explorar el componente actitudinal de los maestros y comenzar a explorar en realidad, el estado actual de la preparación de los maestros para trabajar con los educandos con TEA matriculados en las aulas inclusivas.

En consonancia con el descrito anteriormente, el objetivo de este trabajo es describir y comparar la preparación de los maestros de primaria y secundaria para estimular la socialización de los educandos con TEA en aulas inclusivas en una provincia occidental de Cuba. Los resultados alcanzados podrían servir de base para la elaboración y ejecución de estrategias educativas que contribuyan a potenciar la preparación de los maestros, permitiéndoles tener un papel decisivo y más efectivo en la atención y educación de los educandos con TEA.

La actualidad de la investigación se justifica debido a la escasez de investigación relacionada con la preparación de los maestros para estimular la socialización de los educandos con TEA, al menos en el escenario educacional cubano. Y además en su respuesta a las necesidades de 
la educación actual, la cual requiere de la creación de condiciones para la equiparación de oportunidades y la preparación de los maestros para la atención a la diversidad en las salas de clases inclusivas (Ainscow et al., 2006; Ainscow, 2015; Demósthene, 2010; Pit-ten Cate et al., 2018).

\section{Metodología}

\subsection{Participantes}

Esta investigación se llevó a cabo sobre la base de procedimientos de naturaleza cuantitativa (Dancey \& Reidy, 2017). En consecuencia, se realizó un estudio descriptivo-comparativo de corte transversal, en el cual se seleccionó de manera no probabilística (Intencional) a 74 maestros (52 mujeres y 22 hombres) encargados de cumplir con el rol docente-educativo de atender a los educandos con Trastornos del Espectro Autista en condiciones de inclusión en una provincia occidental de Cuba. El tamaño y las características sociodemográficas de la muestra de estudio se observan en la (Tabla 1).

\section{Tabla 1}

Características sociodemográficas de los maestros estudiados

\begin{tabular}{|c|c|c|c|}
\hline \multicolumn{2}{|c|}{ Variables sociodemográficas } & \multirow{3}{*}{$\begin{array}{c}\mathbf{f i} \\
22 \\
52\end{array}$} & \multirow{3}{*}{$\begin{array}{c}\% \\
29,7 \\
70,3 \\
\end{array}$} \\
\hline$S_{3}$ & Hombres & & \\
\hline Sexo & Mujeres & & \\
\hline \multirow{4}{*}{ Edad } & 22 a 31 años & 31 & 41,9 \\
\hline & 32 a 40 años & 17 & 23 \\
\hline & 41 a 49 años & 19 & 25,7 \\
\hline & 50 a 57 años & 7 & 9,5 \\
\hline \multirow{2}{*}{ Grado Científico } & Pregrado & 60 & 81,1 \\
\hline & Postgrado & 14 & 18,9 \\
\hline \multirow{4}{*}{ Experiencia en la Educación General } & 7 a 110 meses & 31 & 41,9 \\
\hline & 111 a 213 meses & 15 & 20,3 \\
\hline & 214 a 316 meses & 19 & 25,7 \\
\hline & 317 a 420 meses & 9 & 12,2 \\
\hline \multirow{4}{*}{ Experiencia en la Educación Inclusiva } & 2 a 29 meses & 47 & 63,5 \\
\hline & 30 a 55 meses & 12 & 16,2 \\
\hline & 56 a 81 meses & 7 & 9,4 \\
\hline & 82 a 108 meses & 8 & 10,8 \\
\hline \multirow{5}{*}{ Área Disciplinar } & Ciencias Formales & 9 & 12,2 \\
\hline & Ciencias Naturales & 4 & 5,4 \\
\hline & $\begin{array}{c}\text { Ciencias de la Compu- } \\
\text { tación }\end{array}$ & 11 & 14,9 \\
\hline & Ciencias Sociales & 36 & 48,6 \\
\hline & Educación Artística & 14 & 18,9 \\
\hline
\end{tabular}


Se incluyó en el estudio a los maestros que tenían la misión de atender a los educandos con Trastornos del Espectro Autista el sector educacional inclusivo en una provincia occidental de Cuba, y que firmaron la ficha del consentimiento informado. Por otra parte, fueron excluidos los maestros que cumplían con el rol docente de atender a educandos con Trastornos del Espectro Autista, pero que no estaban emplazados en la región seleccionada como objeto de estudio. También los maestros que estaban relacionados con la atención integral de los educandos con necesidades educativas especiales en condiciones de inclusión, pero que no tenían rasgos de autismo y a los que no autorizaron la carta del consentimiento informado.

\subsection{Procedimientos}

Los instrumentos aplicados se construyeron a partir de la operacionalización de la variable, lo cual se separó en dimensiones, indicadores y sub-indicadores (validez de contenido) (ver Tabla 2).

\section{Tabla 2}

\section{Operacionalización de la variable}

\begin{tabular}{|c|c|c|}
\hline Variable & Dimensiones & Indicadores \\
\hline \multirow{5}{*}{$\begin{array}{l}\text { Preparación de los maestros para } \\
\text { estimular la socialización de los } \\
\text { educandos con Trastornos del Es- } \\
\text { pectro Autista en condiciones de } \\
\text { inclusión }\end{array}$} & \multirow[b]{2}{*}{$\begin{array}{l}\text { 1- Preparación Teóri- } \\
\text { ca-Metodológica }\end{array}$} & $\begin{array}{l}\text { Conoce ideas y conceptos acerca de estimular la so- } \\
\text { cialización de los educandos con Trastornos del Es- } \\
\text { pectro Autista en condiciones de inclusión educativa. }\end{array}$ \\
\hline & & $\begin{array}{l}\text { Domina procedimientos metodológicos, recursos, } \\
\text { apoyos y los niveles de ayuda que se emplean en la } \\
\text { estimulación de la socialización de los educandos con } \\
\text { Trastornos del Espectro Autista en condiciones de in- } \\
\text { clusión educativa. }\end{array}$ \\
\hline & \multirow{3}{*}{$\begin{array}{l}\text { 2- Preparación } \\
\text { Práctica }\end{array}$} & $\begin{array}{l}\text { Desarrolla actividades para estimular la socialización } \\
\text { de los educandos con Trastornos del Espectro Autista } \\
\text { en condiciones de inclusión. }\end{array}$ \\
\hline & & $\begin{array}{l}\text { Planifica y aplica de los contenidos académicos en } \\
\text { función de las necesidades y características de los } \\
\text { educandos con Trastornos del Espectro Autista en } \\
\text { condiciones de inclusión. }\end{array}$ \\
\hline & & $\begin{array}{l}\text { Aplica los recursos necesarios para orientar a otros } \\
\text { agentes socializadores y solucionar problemas que } \\
\text { surjan en la atención de los educandos con Trastor- } \\
\text { nos del Espectro Autista en condiciones de inclusión. }\end{array}$ \\
\hline
\end{tabular}

A partir de la operacionalización de la variable se elaboró un cuestionario con 10 preguntas para medir la preparación de los maestros para estimular la socialización de los educandos con TEA en condiciones de inclusión. Se organizó el instrumento en dos partes: (1) datos sociodemográficos de los participantes; (2) información vinculada a la variable de estudio. Se consideraron tres alternativas: 3) pienso que sí, 2) pienso que de forma parcial y 1) pienso que no. El cuestionario diseńado se aplicó a los maestros que firmaron la carta del consentimiento 
informado, de manera individual, en horario laboral durante los meses de febrero y marzo de 2018, en un lugar estratégico orientado por la dirección de las instalaciones educativas seleccionadas, entre las 8:00 a 16:00 horas de lunes a viernes. El proceso transcurrió de manera dialógica, donde los indicadores contemplados en el instrumento se abordaron de manera explicativa en dependencia de la dinámica conversacional. La aplicación de esta herramienta metodológica estuvo a cargo del investigador responsable de la investigación y cumplió con los mismos criterios estándares para todos los participantes.

Asimismo, se elaboró una Guía de Observación de 8 ítems con el objetivo de valorar la preparación de los maestros en la práctica. La guía fue aplicada por el investigador responsable del estudio sin involucrarse en las actividades, es decir de manera no participante. Los ítems de la Guía de observación Las elecciones de medida utilizadas fueron: 3) Siempre, 2) A veces y 1) Nunca. Se aplicó durante la misma secuencia temporal descrita anteriormente, en clases teóricas, prácticas y talleres, por ser formas de organización en las que los maestros demuestran el desarrollo alcanzado en su preparación para estimular la socialización de los educandos comprometidos con esta condición neurobiológica.

La validez de contenido de los instrumentos se determinó por medio del juicio de expertos, mediante el coeficiente $\mathrm{V}$ de Aiken. En ambos instrumentos se obtuvo un amplio grado de acuerdo entre los jueces con intervalos de confianza superiores a 0.60 , excepto en un ítem referido a la Guía de Observación, que se procedió a eliminar. La selección de los expertos se realizó mediante muestreo experto (expert sampling) (Singh, 2007). Se seleccionaron 7 académicos con un alto grado científico y una amplia experiencia en el campo de las Ciencias de la Educación y la Psicología Especial en la Universidad de Matanzas (Cuba), los cuales tuvieron que realizar una valoración conceptual del grado de representatividad, relevancia, diversidad, claridad, sencillez y comprensibilidad de los ítems de los instrumentos. Los puntajes del cuestionario y de la guía de observación oscilaron entre 9 puntos como mínimo y 27 puntos como máximo. Por otra parte, para la estimación de la confiabilidad de los instrumentos se aplicó el coeficiente omega ( $\omega)$. En el cuestionario el coeficiente omega $(\omega)$ mostró un valor de 0,76 y en la Guía de Observación un valor de 0,71 . Por lo tanto, los instrumentos mostraron valores de confiabilidad adecuados en el campo de las ciencias de la educación (Ventura \& Caycho, 2017).

\subsection{ANÁLISIS DE DATOS}

El procesamiento estadístico de los resultados obtenidos de la aplicación de los instrumentos se desarrolló utilizando el programa estadístico SPSS 18.0 y planillas de Excel 2016. Los datos se analizaron por medio de estadística descriptiva: frecuencia (fi), porcentaje (\%), promedio (x) y desviación estándar (DE). Se verificó la normalidad de los datos por el Test de Kolmogorov-Smirnov (K-S). Las diferencias significativas entre sexos y grado científico se verificaron por medio de la prueba t de Student para muestras independientes, asimismo para determinar las diferencias significativas entre grupos de edad cronológicas, experiencia en la educación general, experiencia en la educación especial y áreas disciplinares se utilizó Anova de un factor. La prueba de especificidad de Scheffé se utilizó para verificar las diferencias múltiples. En todos los casos se adoptó una probabilidad de $\mathrm{p}<0.05$. 


\subsection{Aspectos Éticos}

El estudio contó con la aprobación del Comité de Ética de la Universidad Católica del Maule (Acta No: 29), emitida el día 02 de febrero de 2018. Todos los que participaron en el estudio firmaron de manera libre y voluntaria el consentimiento informado.

\section{Resultados Y Discusión}

Los resultados del estudio permitieron reconocer que un significativo porcentaje de los maestros se emplazan con su opinión en el margen de la inconsistencia o tienen una nula capacidad teórica-metodológica y práctica para estimular adecuadamente la socialización de los educandos que presentan alteraciones cualitativas en su neurodesarrollo, incluidos en contextos de aprendizajes inclusivos.

Desde una perspectiva inclusiva es importante que los maestros dominen las bases explicativas del TEA (Hendricks, 2011; Yasar \& Cronin, 2014). Al respecto, la mayor parte de los maestros $(56.8 \%)$ indica que no conoce las características centrales del espectro autista. Asimismo, un 31.1\% revela conocer de manera parcial algunas características y el $12.2 \%$ restante opina que si las conoce.

Para avanzar hacia una educación inclusiva los maestros deben ser capaces de adecuar de manera efectiva sus estrategias pedagógicas a las características particulares de los educandos con TEA (Lindsay et al., 2014). En relación a ello, un $25.7 \%$ considera que no maneja las estrategias metodológicas que se utilizan para trabajar con los educandos con esta condición del neurodesarrollo. Por otro lado, un 39.2\% opina conocerlas de manera parcial y el 35.1\% restante si afirma conocer que estrategias se utilizan para trabajar con estos niños.

El maestro deberá responder a las demandas de cada uno de sus estudiantes, lo cual exige una buena preparación que le permita no solo saber, sino saber hacer (Aguiar et al., 2018; Enríquez et al., 2015). Al respecto, el 33.8\% de los maestros considera que no cuenta con los recursos necesarios para adaptar los contenidos de la asignatura en función de las características de los educandos con TEA. Por otro lado, un grupo más grande de maestros (41.9\%) estima que a veces cuenta con esos recursos pedagógicos y el $24.3 \%$ restante opina que sí.

Promover la participación de los estudiantes con necesidades educativas especiales también es un elemento clave para la inclusión (Echeita, 2013; Lilley, 2015; Yeo \& Teng, 2015). Al respecto, el 24.3\% de los maestros no desarrolla en la sala de clase actividades que estimulen la comunicación y participación de los educandos con TEA. Asimismo, el 37.8\% describe que a veces realiza actividades de estas características y otra proporción de maestros (37.8\%) dice hacerlo con sistematicidad.

Los educandos con TEA son más propensos a presentar dificultades socioemocionales, lo cual constituye un desafío para el maestro dentro del aula. (Marsh et al., 2017; Ratcliffe et al., 2014). Al respecto, un número elevado de maestros (43.2\%) percibe no estar preparados para lidiar con las dificultades socioemocionales de los educandos con autismo en condiciones de inclusión. Por otro lado, el 28.4\% de los maestros estima estar capacitado para hacerlo en ciertas circunstancias y otro $28.4 \%$ opina sí estar preparado. 
La colaboración de los maestros y los padres apoya la enseñanza y favorece la participación y socialización de todos los educandos en condiciones de inclusión (Majoko 2016,2019). En relación a ello, la mayoría de los maestros (50\%) informan sentirse con problemas para orientar y colaborar con los padres de los estudiantes con espectro autista. Sin embargo, el $29.7 \%$ percibe hacerlo en ocasiones y una proporción más pequeńa de maestros (20.3\%) si considera que lo hace de manera constante.

Los participantes describieron una amplia gama de problemas en su preparación teórica-metodológica y práctica para estimular la socialización de los educandos con TEA en escuelas inclusivas. Estos hallazgos son consistentes con otros estudios realizados en el plano internacional, los cuales también han encontrado que existen carencias en la actividad que realizan los maestros para favorecer el desarrollo personal y social de los educandos con necesidades educativas especiales en condiciones de inclusión (Adams et al., 2019; Bryant et al., 2019; Carrington et al., 2016; Santos et al., 2016).

Esto puede pasar porque los maestros que conducen el proceso de enseńanza-aprendizaje en las aulas convencionales no se apropiaron del arsenal teórico y metodológico pertinente en su formación inicial y no tienen la experiencia docente para enfrentar los retos y embates de una misión educativa con estas características (Chkout, et al., 2011; Coady et al., 2016; Tiwari, et al., 2015).

Por otra parte, las comparaciones en la preparación de los maestros por sexo, edad, grado académico, experiencia en la educación general, experiencia en la educación inclusiva y área disciplinar utilizando los datos del cuestionario se observan en la Tabla 3. Se verificaron diferencias significativas entre los maestros participantes cuando se comparó los valores promedios de su preparación teórica-metodológica y práctica según la experiencia en la educación inclusiva y área disciplinar $(\mathrm{p}<0.05)$.

\section{Tabla 3}

Comparación de la preparación teórica-metodológica y práctica de los maestros según los puntajes del cuestionario

\begin{tabular}{l|c|c|c}
\hline \multicolumn{1}{c|}{ Variables sociodemográficas } & $\mathbf{f}$ & $\mathbf{X} \pm \mathbf{D E}$ & $\mathbf{X} \pm \mathbf{D E}$ \\
\hline Sexo & 22 & $7.73 \pm 1.77$ & $11.0 \pm 2.83$ \\
a) Masculino & 52 & $7.33 \pm 2.12$ & $11.3 \pm 3.38$ \\
b) Femenino & & & \\
\hline Edad & 31 & $6.71 \pm 1.71$ & $11.7 \pm 3.17$ \\
a) De 22 a 31 años & 17 & $7.94 \pm 1.91$ & $13.0 \pm 2.64$ \\
b) De 32 a 40 años & 19 & $7.95 \pm 2.29$ & $12.0 \pm 4.83$ \\
c) De 41 a 49 años & 7 & $8.14 \pm 2.11$ & \\
c) De 50 a 57 años & & &
\end{tabular}




\begin{tabular}{|c|c|c|c|}
\hline a) Pregrado & 60 & $7.10 \pm 1.94$ & $10.8 \pm 2.85$ \\
\hline b) Postgrado & 14 & $8.93 \pm 1.68$ & $13.2 \pm 3.84$ \\
\hline \multicolumn{4}{|l|}{$\begin{array}{l}\text { Experiencia en la Educación } \\
\text { General }\end{array}$} \\
\hline a) De 7 a 110 meses & 31 & $6.71 \pm 1.76$ & $9.81 \pm 2.45$ \\
\hline b) De 111 a 213 meses & 15 & $8.20 \pm 1.74$ & $12.0 \pm 3.21$ \\
\hline c) De 214 a 316 meses & 19 & $8.11 \pm 2.18$ & $12.7 \pm 2.90$ \\
\hline d) De 317 a 420 meses & 9 & $7.33 \pm 2.44$ & $12.0 \pm 4.18$ \\
\hline \multicolumn{4}{|l|}{$\begin{array}{l}\text { Experiencia en la Educación } \\
\text { Inclusiva }\end{array}$} \\
\hline a) De 2 a 29 meses & 47 & $6.98 \pm 1.83$ & $10.3 \pm 2.72$ \\
\hline b) De 30 a 55 meses & 12 & $8.75 \pm 1.65$ & $13.2 \pm 3.41^{*}$ \\
\hline c) De 56 a 81 meses & 7 & $7.43 \pm 1.90$ & $11.1 \pm 4.10$ \\
\hline b) De 82 a 108 meses & 8 & $8.25 \pm 2.81$ & $13.8 \pm 2.03^{*}$ \\
\hline \multicolumn{4}{|l|}{ Área Disciplinar } \\
\hline a) Ciencias Formales & 9 & $6.00 \pm 1.50$ & $8.89 \pm 1.61$ \\
\hline b) Ciencias Naturales & 4 & $8.00 \pm 2.16$ & $10.7 \pm 4.19$ \\
\hline c) Ciencias de la Computación & 11 & $7.00 \pm 2.72$ & $10.6 \pm 3.50$ \\
\hline d) Ciencias Sociales & 36 & $7.50 \pm 1.92$ & $11.7 \pm 3.03$ \\
\hline c) Educación Artística & 14 & $8.43 \pm 1.45$ & $12.3 \pm 3.27^{* *}$ \\
\hline
\end{tabular}

Nota: X: promedio, DE: Desviación estándar, ${ }^{*}=$ Diferencia significativa en relación al grupo de 2 a 29 meses dentro de la dimensión práctica $(\mathrm{p}<0,05) ;{ }^{* *}=$ diferencias significativas en relación al grupo de ciencias formales dentro de la dimensión práctica $(\mathrm{p}>0,05)$.

En la Tabla 4 utilizando los datos de la guía de observación se muestran las comparaciones de la preparación teórica-metodológica y práctica de los maestros por sexo, edad, grado académico, experiencia en la educación general, experiencia en la educación inclusiva y área disciplinar. Los resultados muestran de forma general que hubo diferencias significativas entre los maestros en los conocimientos teóricos-metodológicos cuando se comparó por el grado académico y también en su desempeño práctico en la experiencia en la educación inclusiva y área disciplinar $(\mathrm{p}<0.05)$. 


\section{Tabla 4}

Comparación de la preparación teórica-metodológica y práctica de los maestros según los puntajes de la guía de observación

\begin{tabular}{|c|c|c|c|}
\hline Variables sociodemográficas & f & $\begin{array}{c}\text { Preparación Teórica- } \\
\text { Metodológica } \\
\mathrm{X} \pm \mathrm{DE}\end{array}$ & $\begin{array}{c}\text { Preparación } \\
\text { Práctica } \\
\mathrm{X} \pm \mathrm{DE}\end{array}$ \\
\hline $\begin{array}{l}\text { Sexo } \\
\text { a) Masculino } \\
\text { b) Femenino } \\
\end{array}$ & $\begin{array}{l}22 \\
52\end{array}$ & $\begin{array}{l}5.27 \pm 1.80 \\
5.12 \pm 1.52\end{array}$ & $\begin{array}{l}8.59 \pm 2.34 \\
9.40 \pm 2.68\end{array}$ \\
\hline $\begin{array}{l}\underline{\text { Edad }} \\
\text { a) De } 22 \text { a } 31 \text { años }\end{array}$ & 31 & $4.65 \pm 1.33$ & $8.32 \pm 2.21$ \\
\hline b) De 32 a 40 años & 17 & $5.24 \pm 1.30$ & $9.76 \pm 2.10$ \\
\hline c) De 41 a 49 años & 19 & $5.89 \pm 1.96$ & $10.1 \pm 3.25$ \\
\hline c) De 50 a 57 años & 7 & $5.29 \pm 1.79$ & $8.71 \pm 2.43$ \\
\hline $\begin{array}{l}\text { Grado Académico } \\
\text { a) Pregrado }\end{array}$ & 60 & $4.90 \pm 1.36$ & $8.75 \pm 2.35$ \\
\hline b) Postgrado & 14 & $6.29 \pm 2.09^{\mathrm{a}}$ & $10.9 \pm 2.92$ \\
\hline $\begin{array}{l}\text { Experiencia en la Educación General } \\
\text { a) De } 7 \text { a } 110 \text { meses }\end{array}$ & 31 & $4.65 \pm 1.33$ & $8.32 \pm 2.21$ \\
\hline b) De 111 a 213 meses & 15 & $5.33 \pm 1.23$ & $9.73 \pm 2.12$ \\
\hline c) De 214 a 316 meses & 19 & $5.79 \pm 2.01$ & $10.1 \pm 3.16$ \\
\hline d) De 317 a 420 meses & 9 & $5.33 \pm 1.73$ & $9.00 \pm 2.46$ \\
\hline $\begin{array}{l}\text { Experiencia en la Educación Inclusiva } \\
\text { a) De } 2 \text { a } 29 \text { meses }\end{array}$ & 47 & $4.53 \pm 1.12$ & $8.15 \pm 2.00$ \\
\hline b) De 30 a 55 meses & 12 & $6.33 \pm 1.72$ & $11.8 \pm 2.03^{*}$ \\
\hline c) De 56 a 81 meses & 7 & $5.43 \pm 1.71$ & $8.86 \pm 2.67^{*}$ \\
\hline b) De 82 a 108 meses & 8 & $6.88 \pm 1.72$ & $11.3 \pm 2.56^{*}$ \\
\hline $\begin{array}{l}\text { Área Disciplinar } \\
\text { a) Ciencias Formales }\end{array}$ & 9 & $4.11 \pm 1.26$ & $8.67 \pm 1.93$ \\
\hline b) Ciencias Naturales & 4 & $5.50 \pm 1.29$ & $9.25 \pm 2.63$ \\
\hline c) Ciencias de la Computación & 11 & $4.89 \pm 1.26$ & $8.53 \pm 2.36$ \\
\hline d) Ciencias Sociales & 36 & $5.64 \pm 2.11$ & $9.82 \pm 3.51$ \\
\hline c) Educación Artística & 14 & $6.07 \pm 1.81$ & $10.5 \pm 2.37^{\mathrm{b}}$ \\
\hline
\end{tabular}

Nota. X: promedio, DE: Desviación estándar, ${ }^{a}=$ Diferencia significativa en relación al grupo de pre- 
grado dentro de la dimensión teórica-metodológica; ${ }^{*}=$ Diferencia significativa en relación al grupo de 2 a 29 meses dentro de la dimensión práctica; ${ }^{* *}=$ Diferencia significativa en relación al grupo de 30 a 55 meses dentro de la dimensión práctica; ${ }^{b}=$ Diferencia significativa en relación al grupo de ciencias formales dentro de la dimensión práctica.

Los resultados mostraron que cuando se comparó por grado académico, experiencia en la educación inclusiva y área disciplinar, sí hubo diferencias significativas en la preparación teórica-metodológica y práctica de los maestros. Los participantes que tuvieron cursos de postgrado en temas de discapacidad y experiencias de enseñanza en ambientes educativos inclusivos presentaron niveles más altos en la dimensión práctica que los participantes que no tenían o que tenían poca experiencia. Esto pudiera explicarse de acuerdo con lo planteado por Solís et al. (2016), por la falta de preparación de los maestros en la formación inicial en áreas específicas de la educación inclusiva. Estos autores también hacen hincapié en que la actualización por parte de los maestros contribuye a potenciar su capacidad para interactuar con educandos con necesidades educativas especiales. En este sentido, las investigaciones desarrolladas por Bilbao (2008) y Sanz et al. (2017) apuntan en la misma dirección destacando que muchas veces los maestros que trabajan con necesidades educativas especiales en escuelas convencionales tienen falta de formación en temáticas de discapacidad y en metodologías pedagógicas y psicológicas que favorezcan el desarrollo eficaz de prácticas inclusivas.

Estas evidencias concuerdan con los argumentos de Colmenero (2011) y Donnelly \& Watkins (2011), los cuales plantean que los maestros se sienten más capacitados y tienen una percepción más favorable hacia la inclusión cuando se han sometido a espacios de superación posgraduada, lo que repercute en una actitud más favorable hacia los desafíos que son inherentes a un modelo educativo que promueve como norma de desarrollo las prácticas inclusivas. En este sentido, Majoko (2016) también opina que la práctica aplicada en escenarios educativos inclusivos potencia las actitudes positivas y los conocimientos de los maestros, permitiéndoles un mejor manejo del aula y del comportamiento de los educandos con TEA. Esto es un aspecto clave que no se debe pasar por alto en la preparación de los educadores en condiciones del desarrollo social contemporáneo, puesto que existe un consenso de que lo que hacen los maestros en el aula afecta directamente el aprendizaje de los estudiantes (Finkelstein et al., 2019).

En este sentido, Calvo (2013) menciona que hay muchos aspectos por resolver en la preparación de los maestros que continúa anquilosada a la pedagogía convencional y no cuenta con los recursos necesarios para responder de manera adecuada a las problemáticas y desafíos que se presentan en las escuelas inclusivas. Desde esta perspectiva se considera relevante proveer más oportunidades de capacitación que ayuden a los maestros a sentirse más capaces de cumplir con su encargo pedagógico, así como también impulsar durante su formación inicial la participación en ambientes educativos inclusivos (Adams et al., 2017; Srivastava, et al., 2017; Strong, 2014).

Se reconoce que este estudio presenta limitaciones que deben ser señaladas, por ejemplo: la muestra seleccionada es no probabilística y esto se traduce en una limitante de alta consideración que mediatiza la composición de los datos y la posibilidad de generalizar los resultados de la investigación; no se utilizaron otras fuentes información, como la familia , los jefes de ciclo, y los psicopedagogos, los cuales, hubieran podido aportar elementos relevantes para tener una apreciación más global de la preparación de los maestros; y las preguntas del 
cuestionario utilizado podrían haber incluido espacios de proyección abierta que permitieran a los participantes la posibilidad de entregar respuestas más profundas.

La relevancia de este estudio radica en que los directores de escuelas y los maestros podrían desarrollar teniendo en cuenta los resultados, planes de acción y actividades que enriquezcan su labor educativa y potencien la participación de los educandos con TEA. Es necesario que futuras investigaciones consideren estos resultados en sus diseños de investigación y se sugiere seguir investigando este fenómeno, combinando métodos cuantitativos y cualitativos, en otras regiones del país en los cuales los educandos con TEA se encuentren en condiciones de inclusión. Esto será clave para tener una percepción global y holística de la problemática señalada como objeto de estudio.

\section{Conclusiones}

Los resultados del estudio muestran que los maestros de los establecimientos educativos en condiciones de inclusión presentan en proporciones variables dificultades en su preparación teórica-metodológica y práctica para estimular la socialización de los educandos con TEA en escuelas inclusivas. El grado académico, la experiencia en la educación inclusiva y el área disciplinar resultaron variables determinantes en la preparación teórica-metodológica y las habilidades prácticas que éstos poseen para potenciar el desarrollo personal y social de los educandos con esta condición del neurodesarrollo en ambientes escolares inclusivos.

Estos hallazgos sugieren que la participación de los educadores en actividades posgraduales incrementa el manejo de las bases explicativas del trastorno, así como también la experiencia en ambientes educativos inclusivos les ofrece mejores oportunidades de adquirir los recursos psicológicos y pedagógicos necesarios para asumir con mayor seguridad y orientación un encargo educativo de estas características. Esta información debe constituirse en un punto de partida para reflexionar sobre el estado actual de la preparación teórica-metodológica y práctica de los maestros en el sector educacional cubano, aunque para comprobar estos resultados, es necesario desarrollar más estudios en otras zonas del país.

Los resultados sugieren la necesidad de un plan de acción para potenciar la preparación de los maestros, que les permita actualizarse respecto de los adelantos que se producen en las ciencias de la educación abordando la problemática del autismo. Se necesita investigación para explorar el impacto que puede tener la preparación de los maestros en el comportamiento de los estudiantes con TEA en las escuelas inclusivas.

\section{REFERENCIAS}

Adams, M., Rodriguez, S., \& Zimmer, K. (Eds). (2017). Culturally relevant teaching: Preparing teachers to include all learners. Rowman \& Littlefield.

Adams, D., MacDonald, L., \& Keen, D. (2019). Teacher responses to anxiety-related behaviours in students on the autism spectrum. Research in developmental disabilities, 86 (4), 11-19.

Aguiar, G., García, O., Torres, Y., \& Demósthene, Y. (2018). La preparación del maestro para el desarrollo de la comprensión textual en escolares con autismo. Mendive 16(3), 351-364.

Ainscow, M., Booth, T., \& Dyson, A. (2006). Improving Schools, Developing inclusion. Routledge. 
Ainscow, M. (2015). The Index for Inclusion. In Struggles for Equity in Education. Routledge.

American Psychiatric Association (2013). Diagnostic and statistical manual of mental disorders (5th ed.). American Psychiatric Publishing.

Anglim, J., Prendeville, P., \& Kinsella, W. (2018). The self-efficacy of primary teachers in supporting the inclusion of children with autism spectrum disorder. Educational Psychology in Practice, 34(1), 73-88. https://doi.org/10.1080/02667363.2017.1391750

Ashburner, J., Ziviani, J., \& Rodger, S. (2010). Surviving in the mainstream: Capacity of children with autism spectrum disorders to perform academically and regulate their emotions and behavior at school. Research in Autism Spectrum Disorders, 4(1), 18-27.

Bilbao, C. (2008). La integración de personas con discapacidad en la Educación Superior. Percepciones y demandas docentes y estudiantes de la Universidad de Burgos. [Tesis Doctoral, Universidad de Burgos]. Repositorio UBU.

Bryant, D., Bryant, R., \& Smith, D. (2019). Teaching students with special needs in inclusive classrooms. Sage Publications.

Calvo, G. (2013). La formación de docentes para la inclusión educativa. Páginas de Educación, 6(1), $19-35$.

Campos, I. (2010). Una estrategia de educación familiar en la escuela para niños con autismo. [Tesis de Doctorado, Universidad de la Habana]. Repositorio Universidad de la Habana.

Carrington, S., Berthelsen, D., Nickerson, J., Nicholson, J. M., Walker, S., \& Meldrum, K. (2016). Teachers' Experiences of Inclusion of Children with Developmental Disabilities across the Early Years of School. Journal of Psychologists and Counsellors in Schools, 26(02), 139-154. https://doi. org/10.1017/jgc.2016.19

Castillo, T. (2004). La preparación del nuevo maestro. [Tesis de Maestría, Instituto Pedagógico Latinoamericano y del Caribe]. La Habana, Cuba.

Chen, Y. M., Jin, Y., Zhang, L., Wan, B., \& Pan, N. (2017). Understanding and attitudes of primary school teachers on autism spectrum disorders in Guangzhou. Journal of Community Medicine, 7(15), 5. https://en.cnki.com.cn/Article_en/CJFDTotal-SQYX201715005.htm

Christensen, D., Baio, J., Braun, K. V. N., Bilder, D., Charles, J., Constantino, J. N., Daniels, J., Maureen, S. D., Fitzgerald, R. T., Kurzius-Spencer, M., Lee, L.C., Pettygrove, S., Robinson, C., Schulz, E., Wells, C., Wingate, M. S., Zahorodny, W., \& Yeargin-Allsopp, M. (2016). Prevalence and characteristics of autism spectrum disorder among children aged 8 years-autism and developmental disabilities monitoring network, 11 sites, United States, 2012. MMWR Surveillance Summaries, 65(3), 1- 23.

Chkout, C., Sarduy, C., Tunas, L., \& Leyva, C. (2011). Una concepción enriquecedora de atención educativa a los niños con diagnóstico del autismo y sordoceguera. Editorial Pueblo y Educación.

Chung, W., Chung, S., Edgar-Smith, S., Palmer, R. B., DeLambo, D., \& Huang, W. (2015). An examination of in-service teacher attitudes toward students with autism spectrum disorder: Implications for professional practice. Current Issues in Education, 18(2), 1-12.

Coady, M., Harper, C., \& De Jong, J. (2016). Aiming for equity: Preparing mainstream teachers for inclusion or inclusive classrooms? Tesol Quarterly, 50(2), 340-368. 
Colmenero, M. (2011). Factores que inciden y repercuten en los procesos de atención a la diversidad de los docentes de educación secundaria obligatoria. Rev. Int. Investig. Cienc. Soc, 7(1), 61-90.

Dancey, C., \& Reidy, J. (2017). Statistics without maths for psychology. Pearson.

Demósthene, Y. (2010). Un programa educativo para la estimulación del desarrollo de la socialización en los niños con autismo en la primera infancia. [Tesis doctoral, Universidad de la Habana].

Donnelly, V., \& Watkins, A. (2011). Teacher education for inclusion in Europe. Prospects, 41, 341-353. https://doi.org/10.1007/s11125-011-9199-1

Echeita, G. (2013). El apoyo entre el alumnado para aprender y participar. En G. Echeita, C. Simón, M. Sandoval, \& H. Monarca (Eds.), Cómo fomentar las redes naturales de apoyo en el marco de una escuela inclusiva. Propuestas prácticas (pp. 55-76). MAD.

Enríquez, C., Navarro, G., \& Ochoa, C. (2015). La evaluación psicopedagógica de niños (as) con trastornos del espectro autista mediante el perfil psicoeducativo. Universidad y Sociedad, 7(2). 1415.

Finkelstein, S., Sharma, U., \& Furlonger, B. (2019). The inclusive practices of classroom teachers: a scoping review and thematic analysis. International Journal of Inclusive Education, 1-28. https:// doi.org/10.1080/13603116.2019.1572232

Fombonne, E. (2018). The rising prevalence of autism. Journal of Child Psychology and Psychiatry, 59(7), 717-720.

Garcés, R., \& Zambrano, M. (2018). Actitudes de los docentes hacia la inclusión escolar de niños con autismo. Killkana sociales: Revista de Investigación Científica, 2(4), 39-48.

García, X., Massani, J., \& Bermúdez, I. (2016). La educación inclusiva en la formación de profesionales de la educación. Revista Universidad y Sociedad, 8(1), 118-121.

Garrad, T., Rayner, C., \& Pedersen, S. (2018). Attitudes of Australian primary school teachers towards the inclusion of students with autism spectrum disorders. Journal of Research in Special Educational Needs, 19(1), 58-67, https://doi.org/10.1111/1471-3802.1242

Hendricks, D. (2011). Special education teachers serving students with autism: A descriptive study of the characteristics and self-reported knowledge and practices employed. Journal of Vocational Rehabilitation, 35(1), 37-50.

Lilley, R. (2015). Trading places: Autism inclusion disorder and school change. International Journal of Inclusive Education, 19(4), 379-396.

Lindsay, S., Proulx, M., Thomson, N., \& Scott, H. (2013). Educators' Challenges of Including Children with Autism Spectrum Disorder in Mainstream Classrooms. International Journal of Disability, Development and Education, 60(4), 347-362. https://doi.org/10.1080/1034912x.2013.846470

Lindsay, S., Proulx, M., Scott, H., \& Thomson, N. (2014). Exploring teachers' strategies for including children with autism spectrum disorder in mainstream classrooms. International Journal of Inclusive Education, 18(2), 101-122.

Low, H., Lee, L., \& Che Ahmad, A. (2018). Pre-service teachers' attitude towards inclusive education for students with autism spectrum disorder in Malaysia. International Journal of Inclusive Education, 22(3), 235-251.

Lozic, V. (2014). Inclusion through exclusion: teachers' perspectives on teaching students with autism. Cypriot Journal of Educational Sciences, 9(1), 3-13. 
Majoko, T. (2016). Inclusion of children with autism spectrum disorders: Listening and hearing to voices from the grassroots. Journal of autism and developmental disorders, 46(4), 1429-1440.

Majoko, T. (2019). Inclusion of children with autism spectrum disorder in mainstream early childhood development: Zimbabwean parent perspectives. Early Child Development and Care, 189(6), 909925

Marsh, A., Spagnol, V., Grove, R., \& Eapen, V. (2017). Transition to school for children with autism spectrum disorder: A systematic review. World journal of psychiatry, 7(3), 184.

McCurdy, E., \& Cole, L. (2013). Use of a Peer Support Intervention for Promoting Academic Engagement of Students with Autism in General Education Settings. Journal of Autism and Developmental Disorders, 44(4), 883-893. https://doi.org/10.1007/s10803-013-1941-5

Organización de las Naciones Unidas para la Educación, la Ciencia y la Cultura (1990). Declaración mundial sobre educación para todos y Marco de acción para satisfacer las necesidades básicas de aprendizaje. Jomtien: UNESCO. https://unesdoc.unesco.org/ark:/48223/pf0000184556

Organización de las Naciones Unidas para la Educación, la Ciencia y la Cultura (1994). La Declaración de Salamanca y el Marco de acción sobre la educación para necesidades especiales. Paris: UNESCO.

Organización de las Naciones Unidas (2006). Convención sobre los derechos de las personas con discapacidad y protocolo facultativo. New York: Naciones Unidas.

Pit-ten Cate, I., Markova, M., Krischler, M., \& Krolak-Schwerdt, S. (2018). Promoting Inclusive Education: The Role of Teachers' Competence and Attitudes. Insights into Learning Disabilities, 15(1), 49-63.

Prizant, B. (2015). Uniquely Human: A Different Way of Seeing Autism. Simon \& Schuster.

Ratcliffe, B., Wong, M., Dossetor, D., \& Hayes, S. (2014). Teaching social-emotional skills to schoolaged children with Autism Spectrum Disorder: A treatment versus control trial in 41 mainstream schools. Research in Autism Spectrum Disorders, 8(12), 1722-1733. https://doi.org/10.1016/j. rasd.2014.09.010

Roberts, J., \& Simpson, K. (2016). A review of research into stakeholder perspectives on inclusion of students with autism in mainstream schools. International Journal of Inclusive Education, 20(10), 1084-1096. https://doi.org/10.1080/13603116.2016.1145267

Rodden, B., Prendeville, P., Burke, S., \& Kinsella, W. (2019). Framing secondary teachers' perspectives on the inclusion of students with autism spectrum disorder using critical discourse analysis. Cambridge Journal of Education, 49(2), 235-253.

Santos, G., Sardinha, S., \& Reis, S. (2016). Relationships in inclusive classrooms. Journal of Research in Special Educational Needs, 16(3), 950-954.

Sanz, P., Fernández, M., Pastor, G., \& Tárraga, R. (2017). Pre-ServiceTeachers' Knowledge, Misconceptions and Gaps About Autism Spectrum Disorder. The Journal of the Teacher Education Division of the Council for Exceptional Children, 40(3), 212-224. https://doi.org/10.1177/0888406417700963

Serrano, E. A. P. (2017). La inclusión como un proceso por el sistema educativo: experiencias de inclusión en la universidad de Holguín, CUBA. Educação \& Sociedade, 38(138), 81-98. https:// doi.org/10.1590/es0101-73302017151507

Singh, K. (2007). Quantitative Social Research Methods. Sage Publications. 
Smiley, K., Gerstein, B., \& Nelson, S. (2018). Unveiling the autism epidemic. Journal of Neurology and Clinical Neuroscience, 2(2), 1.

Snyder, T., \& S, Dillow. (2013). Digest of Education Statistics (NCES 2014-015). Jessup, PA: National Center for Education Statistics, Institute of Education Science

Solís, C., Núñez, C., Vásquez, N., Contreras, I., y Ritterhaussen, S. (2016). Problemas en la inserción profesional de profesores: necesidad de mentoría. Estudios pedagógicos (Valdivia), 42(4), 201-221. https://doi.org/10.4067/S0718-07052016000500012

Soto-Chodiman, R., Pooley, J. A., Cohen, L., \& Taylor, M. F. (2012). Students with ASD in mainstream primary education settings: Teachers' experiences in Western Australian classrooms. Australasian Journal of Special Education, 36(2), 97-111. https://doi.org/10.1017/jse.2012.10

Strong, J. (2014). Preparing teachers of students with Autism spectrum disorders: Evidence-based practices and teacher self-efficacy. [PhD dissertation, Commonwealth University]. Commonwealth University Repositorio.

Srivastava, M., de Boer, A., \& Pijl, S. (2017). Preparing for the inclusive classroom: changing teachers' attitudes and knowledge. Teacher Development, 21(4), 561-579.

Tiwari, A., Das, A., \& Sharma, M. (2015). Inclusive education a "rhetoric" or "reality"? Teachers' perspectives and beliefs. Teaching and Teacher Education, 52, 128-136. https://doi.org/10.1016/j. tate.2015.09.002

Tromans, S., Chester, V., Kiani, R., Alexander, R., \& Brugha, T. (2018). The Prevalence of Autism Spectrum Disorders in Adult Psychiatric Inpatients: A Systematic Review. Clinical Practice \& Epidemiology in Mental Health, 14(1), 177-187. https://doi.org/10.2174/1745017901814010177

Ventura, J., \& Caycho, T. (2017). El coeficiente Omega: un método alternativo para la estimación de la confiabilidad. Revista Latinoamericana de Ciencias Sociales, Niñez y Juventud, 15(1), 625-627.

Vorstman, J., Parr, R., Moreno, D., Anney, R., Nurnberger Jr, J., \& Hallmayer, J. (2017). Autism genetics: opportunities and challenges for clinical translation. Nature Reviews Genetics, 18(6), 362376. https://doi.org/10.1038/nrg.2017.4

Yasar, P., \& Cronin, K. (2014). Perspectives of College of Education Students in Turkey on Autism Spectrum Disorders. International Journal of Special Education, 29(1), 61-75.

Yeo, J., \& Teng, K. (2015). Social Skills Deficits in Autism: A Study among Students with Austim Spectrum Disorder in Inclusive Classrooms. Universal Journal of Educational Research, 3(12), 10011007.

Recebido em: 26/09/2020

Reformulado em: 29/09/2020

Aprovado em: 17/10/2020 